Abstract

\title{
Identification of New Single Cross Testers for CIMMYT Heterotic Group B Maize Germplasm Adapted to Mid-Altitudes ${ }^{\dagger}$
}

\author{
Arisede Chisaka ${ }^{1,2, *}$, Vivi Arief ${ }^{1}$, Amsal Tarekegne ${ }^{2}$ and Mark Dieters ${ }^{1}$ \\ 1 School of Agriculture and Food Science, The University of Queensland, St Lucia, QLD 4067, Australia; \\ v.arief1@uq.edu.au (V.A.); m.dieters@uq.edu.au (M.D.) \\ 2 International Maize and Wheat Improvement Center (CIMMYT), P.O. Box MP163 Harare, Zimbabwe; \\ a.tarekegne63@gmail.com \\ * Correspondence: arisedechisaka@gmail.com \\ † Presented at the Third International Tropical Agriculture Conference (TROPAG 2019), Brisbane, Australia, \\ 11-13 November 2019.
}

Published: 10 April 2020

\begin{abstract}
The development of higher yielding maize (Zea mays L.) hybrids adapted to low input small-holder farming systems in sub-Saharan Africa is required. Three-way hybrids (i.e. F1 tester crossed to inbred line) can be produced at lower cost for smallholder farmers, but to achieve this it is crucial for CIMMYT-Zimbabwe maize breeding program to identify new high yielding single cross testers with high combining ability to support the development of new high yielding hybrids for this region. Data collected on the performance of F1 and three-way hybrids from a total of 25 multi-environment trials (METs) located in South Africa (three trials), Zambia (four trials) and Zimbabwe (eighteen trials) grown across two seasons during the period from 2015-2018 to: (i) identify new single cross testers for CIMMYT HG-B maize germplasm; (ii) identify stable and high yielding three-way hybrids. Analyses were conducted using a two-stage approach. Clustering based on yield data, grouped sites into three environment types (ET); low (LY) $<3 \mathrm{t} \mathrm{ha}^{-1}$, medium (MY) 3-6 tha-1 and high (HY) 6-13 t ha $\mathrm{a}^{-1}$ yielding groups. Additive genetic effects of both inbred parents and selected F1 crosses used as parents were more important than non-additive genetic effects for grain yield across ETs. Strong genotype $x$ environment interactions on yield and other traits were observed. It was concluded that F1 hybrids (entry 75, 85, 72 and 28) demonstrated high yield across all environment types, and it is recommended that these be also evaluated as potential three-way hybrids. Single crosses CZL15085/CML566 and CZL15085/CZL13102 from heterotic group B to be used as testers for ET 1 and ET 2 respectively.
\end{abstract}

Keywords: maize; single cross; tester; heterotic group; three-way maize hybrids

Author Contributions: A.C. wrote the manuscript, V.A. have made substantial contributions to analysis and interpretation of data, A.T. have given technical advice and manuscript editing and M.D. manuscript editing and technical advice. All authors have read and agreed to the published version of the manuscript.

Acknowledgments: The authors of this report gratefully acknowledge the financial contributions of Bill and Melinda Gates Foundation and USAID CIMMYT to implement this study.

Conflicts of Interest: The authors declare no conflict of interest.

(C) 2020 by the authors. Licensee MDPI, Basel, Switzerland. This article is an open access article distributed under the terms and conditions of the Creative Commons Attribution (CC BY) license (http://creativecommons.org/licenses/by/4.0/). 